\title{
Resonant Characteristics of a Corrugated Sphere
}

\author{
James R. Wait and Carolen M. Jackson \\ Contribution From Central Radio Propagation Laboratory, National Bureau of Standards, \\ Boulder, Colo.
}

(Received December 28, 1962)

\begin{abstract}
The radiation from a radial electric dipole on the surface of a corrugated sphere is treated. It is shown that the power radiated in a given mode depends critically on the surface reactance and the circumference of the sphere. In fact, for certain values of these parameters, particular modes are strongly excited and contain most of the power. Such a structure can be regarded as an external resonator and its resonant characteristics are a function of the refractive index of the surrounding medium. This opens the possibility that a surface-wave spherical resonator may have important applications to refractometry.
\end{abstract}

\section{Introduction}

A corrugated surface on a dielectric-coated plane surface will support a nonradiating surface wave [Zucker, 1961]. In fact, even if the surface is partly curved, it has been shown that the wave excited on the structure bears a close resemblance to that of a lossless plane surface [Wait, 1962]. The curvature of the guiding surface tends to produce some leakage of energy in the normal direction although most of the energy is still guided tangentially around the periphery of the structure. Consequently, a corrugated regular shaped body, which is closed on itself, should be able to support circulating surface waves. For certain critical dimensions it can be expected that some interesting resonant phenomena will result.

The possibility that a closed corrugated cylindrical structure (of infinite length) will exhibit resonance phenomena has been considered on prior occasions [Cullen, 1960; Wait and Conda, 1960]. It is the purpose of the present paper to extend these arguments to a spherical body which has the practical advantage of being a finite structure.

\section{Formulation}

The model chosen is a sphere of radius $a$ which is corrugated by shallow concentric grooves. Choosing a spherical coordinate system $(r, \theta, \phi)$, a radial dipole $I d s$ is located at $r=b$ with respect to the sphere at $r=a$ and, of course, $b \geqq a$. The situation is illustrated in figure 1 . The boundary conditions at the surface of the sphere are

$$
\left.E_{\theta}=-i X H_{\phi}\right]_{r=a}
$$

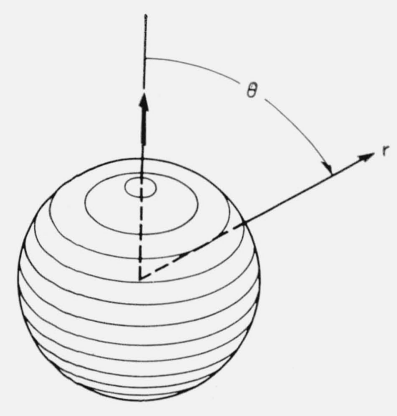

FIGURE 1. The latitudinally corrugated sphere excited by a radicl electric dipole at the pole. 
where $X$ is the surface reactance. If the grooves are rectangular in cross section, with periodicity $d$, width $w$, it is known that [Zucker, 1961]

$$
X \cong \eta_{0} \frac{w}{d} \tan k l
$$

where $\eta_{0}=120 \pi$ and $k=2 \pi /$ wavelength. This formula is valid provided that $k d<<1$ and $k l$ is not near an odd multiple of $\pi / 2$.

For the presently posed problem, symmetry considerations indicate that the resulting field is purely TM (transverse magnetic). Consequently, the individual field components may be derived in terms of a single scalar function $U$ as follows:

$$
\begin{gathered}
E_{r}=\left(k^{2}+\frac{\partial^{2}}{\partial r^{2}}\right)(r U) \\
E_{\theta}=\frac{1}{r} \frac{\partial^{2}}{\partial r \partial \theta}(r U) \\
H_{\phi}=-i \epsilon \omega \frac{\partial U}{\partial \theta}
\end{gathered}
$$

while $E_{\phi}=0$ and $H_{r}=H_{\theta}=0$.

\section{Solution}

The fields of the dipole in the presence of the sphere can be expressed in the form

$$
U=U^{p}+U^{s}
$$

where $U^{p}$ is the primary influence and $U^{s}$ is the secondary influence. Now

$$
U^{p}=-C e^{-i k R} /(4 \pi R)
$$

where $R=\left[r^{2}+b^{2}-2 b r \cos \theta\right]^{1 / 2}$ is the distance from the source to the observer at $(r, \theta)$ and $C=\frac{i I d s}{\epsilon \omega b}$. Making use of an addition theorem for spherical wave functions [Morse and Feshbach, 1953]

$$
U^{p}=\frac{i k C}{4 \pi} \sum_{q=0}^{\infty}(2 q+1) P_{q}(\cos \theta)\left\{\begin{array}{l}
j_{q}(k r) h_{q}^{(2)}(k b) ; \text { for } r<b \\
h_{q}^{(2)}(k r) j_{q}(k b) ; \text { for } r>b
\end{array}\right.
$$

where $j_{q}$ and $h_{q}^{(2)}$ are spherical Hankel functions and $P_{q}(\cos \theta)$ is a Legendre polynomial. Since

$$
\left(\nabla^{2}+k^{2}\right) U^{s}=0
$$

and because $U^{s}$ must be an outgoing wave at infinity, it follows that

where

$$
U^{s}=\frac{i k C}{4 \pi} \sum_{q=0}^{\infty}(2 q+1) P_{q}(\cos \theta) A_{q} h_{q}^{(2)}(k r)
$$

$$
A_{q}=-\frac{j_{q}(k a)}{h_{q}^{(2)}(k a)}\left[\frac{\frac{d}{d x} \log \left[x j_{q}(x)\right]+G}{\frac{d}{d x} \log \left[x h_{q}^{(2)}(x)\right]+G}\right]_{x=k a} h_{q}^{(2)}(k b)
$$

and where $G=X / \eta_{0}$. The veracity of this equation may be confirmed by seeing that it satisfies the required boundary condition at $r=a$.

In order to simplify matters, the source dipole is located on the sphere (i.e., $b=a$ ) and 
the observer is in the far field (i.e., $k r \rightarrow \infty$ ). It then follows without difficulty that

$$
H_{\phi} \cong \frac{I d s}{4 \pi \operatorname{rax}} \sum_{q=0}^{\infty} \frac{(2 q+1) i^{q+1} P_{q}^{1}(\cos \theta)}{\frac{d}{d x}\left[x h_{q}^{(2)}(x)\right]+G\left[x h_{q}^{(2)}(x)\right]} e^{-i k r}
$$

where $x=k a$.

In obtaining this result, use has been made of (5), the Wronskian relation

the asymptotic formula

$$
W\left[x j_{q}(x), x h_{q}^{(2)}(x)\right]=-i,
$$

$$
h_{q}^{(2)}(k r) \sim \frac{1}{k r} i^{(n+1)} e^{-i k r} \text { for } k r \rightarrow \infty,
$$

and the derivative relation

$$
\frac{\partial}{\partial \theta} P_{q}(\cos \theta)=-P_{q}^{1}(\cos \theta)
$$

\section{Power Radiated}

In the present problem, a meaningful quantity is the total power $P$ supplied by the dipole. Since the structure is lossless, $P$ can be obtained by integrating the real Poynting vector over a concentric spherical surface of infinitely large radius. This readily leads to

$$
P=\operatorname{Lim}_{r \rightarrow \infty} \frac{\eta_{0}}{2} \int_{0}^{\pi}\left[H_{\phi} H_{\phi}^{*}\right] 2 \pi r^{2} \sin \theta d \theta
$$

where the asterisk denotes a complex conjugate. To perform the integration, it is noted that

$$
\begin{aligned}
\int_{0}^{\pi} P_{q}^{1}(\cos \theta) P_{q^{\prime}}^{1}(\cos \theta) \sin \theta d \theta & =\int_{-1}^{+1} P_{q}^{1}(z) P_{q^{\prime}}^{1}(z) d z \\
& =0 \text { if } q \neq q^{\prime} \\
& =\frac{2(q+1) q}{2 q+1} \text { if } q=q^{\prime} .
\end{aligned}
$$

The resulting expression for the total power is then

$$
P=\eta_{0} \frac{(I d s)^{2}}{8 \pi a^{2} x^{2}} \sum_{q=0}^{\infty} p_{q}
$$

where

$$
p_{q}=\frac{q(q+1)(2 q+1)}{\left|\frac{d}{d x}\left[x h_{q}^{(2)}(x)\right]+G x h_{q}^{(2)}(x)\right|^{2}} .
$$

This result may be written in more convenient form for calculation if one notes that

$$
h_{q}^{(2)}(x)=j_{q}(x)-i y_{q}(x)
$$

where $j_{q}$ and $y_{q}$ are real. Thus

$$
p_{q}=\frac{q(q+1)(2 q+1)}{\left[(1+G x) j_{q}(x)+x j_{q}^{\prime}(x)\right]^{2}+\left[(1+G x) y_{q}(x)+x y_{q}^{\prime}(x)\right]^{2}}
$$

In some instances it is convenient to define a radiation resistance $R$ such that

$$
P=I^{2} R / 2 \text {. }
$$


Then

where

$$
\frac{R}{R_{0}}=\frac{3}{8 x^{4}} \sum_{q=0}^{\infty} p_{q}
$$

$$
R_{0}=80(k d s)^{2}
$$

is the radiation resistance of the same dipole if it were located in free space.

\section{Alternative Derivation by EMF Method}

The expression for the total power given by (18) can also be found by the so-called "induced emf method" [Carter, 1932]. The procedure is not particularly rigorous but it does lead to correct results. The derivation is outlined very briefly here. The reader should have no difficulty filling in the steps.

By making use of (8) and (9) and the Wronskian relation (13), it follows that, for $b=a$,

where $x=k a$ and

$$
r U=\frac{I d s}{4 \pi i \epsilon \omega a} \sum_{q=0}^{\infty} \frac{(2 q+1) \hat{h}_{q}(k r)}{x\left[\hat{h}_{q}^{\prime}(x)+G \hat{h}_{q}(x)\right]} P_{q}(\cos \theta)
$$

$$
\hat{h}_{q}(x)=x h_{q}^{(2)}(x)
$$

Using (3) and the basic differential equation

it is found that

$$
\left[\frac{d^{2}}{d r^{2}}+k^{2}-\frac{q(q+1)}{r^{2}}\right] \hat{h}_{q}(k r)=0,
$$

$$
E_{r}=\frac{I d s}{4 \pi i \epsilon \omega a r^{2}} \sum_{q=1}^{\infty} \frac{q(q+1)(2 q+1) \hat{h}_{q}(k r)}{x\left[\hat{h}_{q}^{\prime}(x)+G \hat{h}_{q}(x)\right]} P_{q}(\cos \theta) .
$$

An expression for the radiation resistance $R$ is now found from the prescription

$$
R=\operatorname{Re}\left[\frac{E_{r} d s}{I}\right]_{\substack{\theta \rightarrow 0 \\ r \rightarrow a}}
$$

where Re signifies that the real part is to be taken. To effect this limiting process, it is noted that

$$
\operatorname{Re}\left[\frac{-i \hat{h}_{q}(x)}{\hat{h}_{q}(x)+G \hat{h}_{q}(x)}\right]=\frac{1}{\left|\hat{h}_{q}^{\prime}(x)+G \hat{h}_{q}(x)\right|^{2}}
$$

which is valid if $G$ and $x$ are real. The latter identity can be established by making further use of the Wronskian relation (13). The final result for $R$ is identical to (23), which is to be expected.

\section{Discussion of Numerical Results}

To illustrate the resonance phenomena, the function $p_{q}$ is plotted as a function of the normalized surface reactance $G$ for $x=3$ and several values of $q$. The results are shown in figure 2 where the ordinate is the quantity $10 \log _{10} p_{q}$ in decibels. It is evident that each mode has a strong resonance for a particular value of $G$. This resonance occurs when the denominator of (21) passes through a minimum. Because the $y$ functions are much larger than the $j$ functions it turns out th the minimum coincides almost exactly with a zero of the second factor in the denominator. Therefore, the condition for a resonance is

$$
\left[(1+G x) y_{q}(x)+x y_{q}^{\prime}(x)\right] \simeq 0,
$$


or

$$
G=-\frac{1}{x}-\frac{y_{q}^{\prime}(x)}{y_{q}(x)}
$$

It is seen that the resonance peaks in figure 2 become progressively sharper as the order $q$ of the mode increases. Unfortunately, however, the value of the surface reactance to achieve resonance becomes larger. For example, the resonance condition at $q=8$ requires that $G=2.456$ or $X=920$ ohms. It is quite difficult, in a mechanical sense, to achieve a purely inductive reactance of this magnitude with a corrugated structure. On the other hand, the realization of a corrugated sphere to exhibit resonance for the lower order resonance would not be quite so difficult. For example, the resonance at $q=6$ occurs when $G=1.693$.

The influence of a small change of frequency from its resonance value is also of interest. For example, if the relative change of frequency is $\delta$ the value of $k a$ changes from 3 to $3(1+\delta)$. If the sphere is corrugated the inductive reactance is also approximately proportional to frequency. Thus, $G$ changes from 1.693 to $1.693(1+\delta)$. The effect of such a change is illustrated in figure 3. As expected, the shift of frequency will cause a certain amount of "detuning." For a gross change of frequency, the structure will become resonant at another mode. For example, at a 10 percent change of frequency, the mode at $q=7$ has a resonant peak. Actually, for a realistic structure the detuning effect is probably more severe than shown in figure 3, because the frequency dependence of the surface reactance is more pronounced than a linear law.

Of particular importance is the behavior of a resonant peak when the refractive index of the surrounding medium changes. In this case, the surface reactance parameter $G$ can be regarded as a constant but the value of $k a$ changes to $k a(1+\Delta)$ for an incremental refractive index change

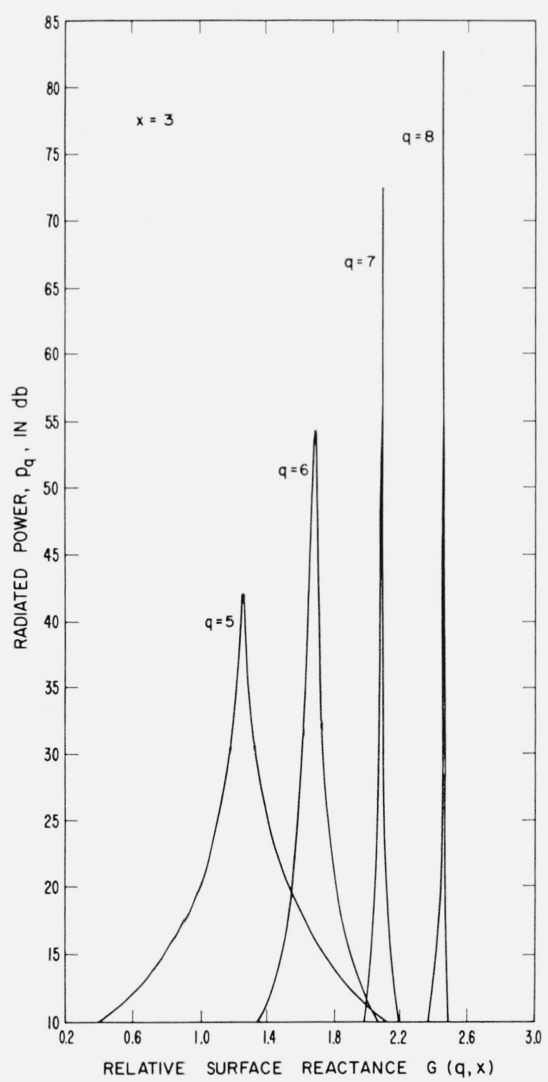

Figure 2. The radiated power $\mathrm{p}_{\alpha}$ in the various modes as a function of the normalized surface reactance for a sphere whose circumference is 3 wavelengths.

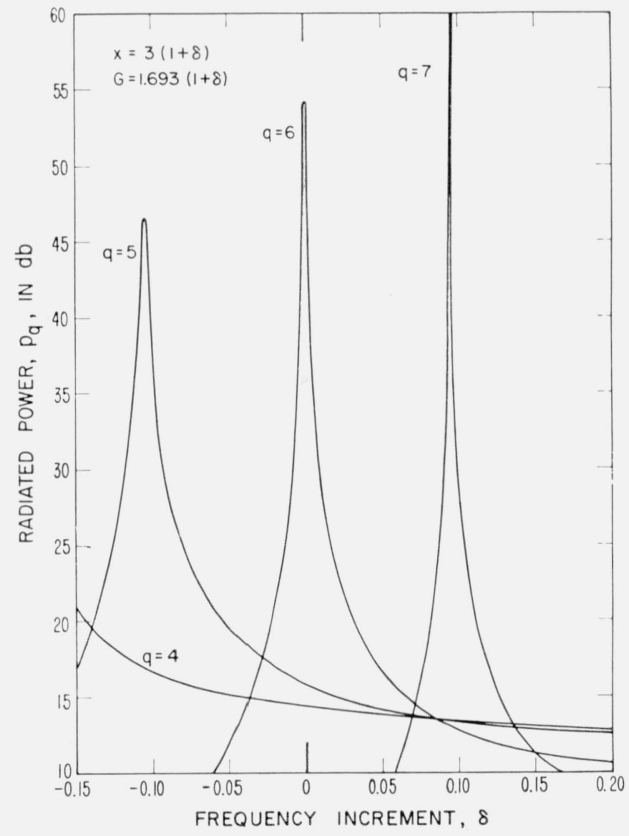

FIGURE 3. The "detuning" effect"of changing" freqency by a fractional amount $\delta$ from resonance for a corrugated sphere. 


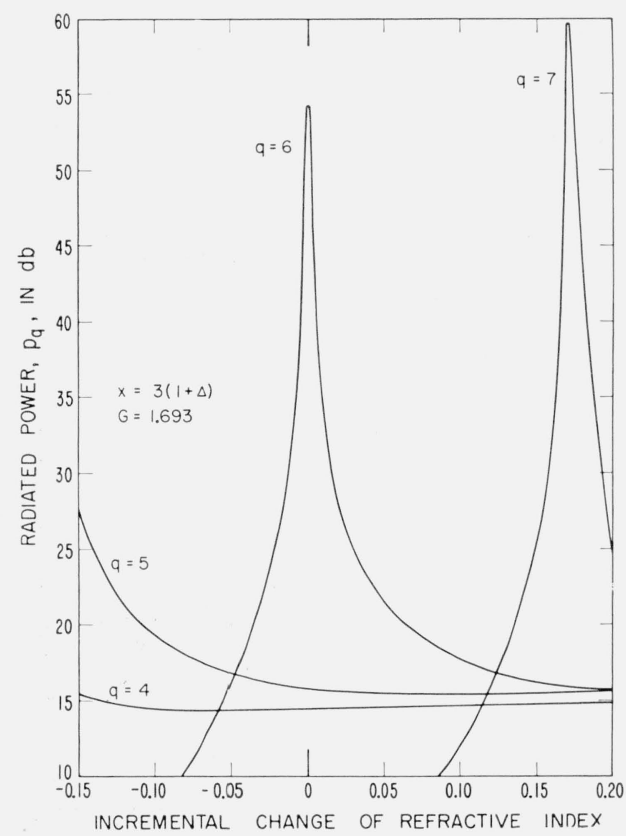

FIGURE 4. The effect of changing the refractive index of the surrounding medium.

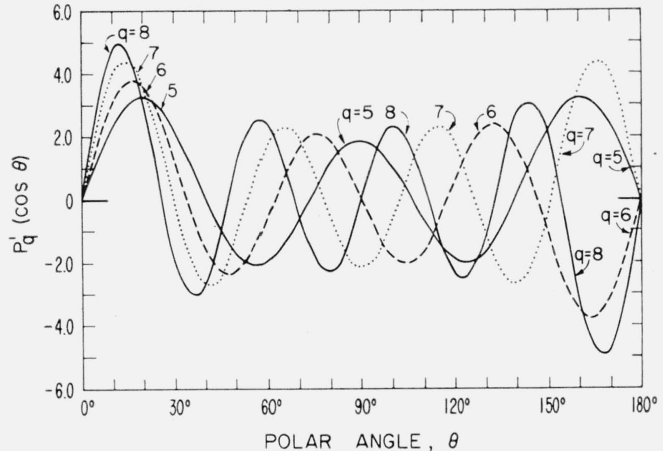

Figure 5. The Legendre polynomial $\mathrm{P}_{\mathrm{q}}^{1}(\cos \theta)$ for

These characterize the radiation patterns for the various modes.

of $\Delta$. The situation is clearly illustrated in figure 4 where $k a$ is again taken to be 3 . The curves in figure 4 apply to the case where the environment changes and, consequently, the resonant sphere could be regarded as a refractometer. The possibility that such an external resonator could be used as a method of measuring the refractive index of various gases should be investigated. The method may have some merit in the probing of plasma from spherical satellites.

In the foregoing discussion attention has been restricted to the radiated power of the various modes. As indicated, the structure may be designed so that most of the power goes into only one mode. The radiation pattern of such a mode is described by the function $P_{q}^{1}(\cos \theta)$ as can be indicated by (12). Although these functions are very well known, their behavior is sketched in figure 5 for convenience of the reader. As indicated, the number of lobes in the radiation pattern increases with the mode number $q$. It is interesting to note that the radiation pattern has a null in the axial directions as expected. Also, it is observed that nulls occur in the broadside direction (i.e., $\theta=90^{\circ}$ ) for the even modes. On the other hand, the even-ordered modes have a lobe maximum in the broadside direction. This fact may be helpful in certain practical schemes which are used to detect the resonances by measuring the radiation pattern.

\section{Comparison With Cylindrical Model}

There are certain aspects of the present problem which must bear close resemblance to the resonance with corrugated cylinders. The cylindrical analog is an axial slot (line magnetic source) on the surface of a longitudinally corrugated cylinder of radius $a_{c}$. Such a mode? was considered in a previous paper [Wait and Conda, 1960] so theoretical details need not be elaborated on. Leaving aside constant factors, it was shown that the total power radiated was given by

where

$$
P_{c}=\sum_{m=0}^{\infty} p_{m}
$$

$$
p_{m}=\frac{\epsilon_{m}}{x^{2}} \frac{1}{\left|H_{m}^{(2) \prime}(x)+G H_{m}^{(2)}(x)\right|^{2}}
$$


Figure 6. The "detuning" effect for a corrugated cylinder shown for purposes of comparison' with figure 3 .

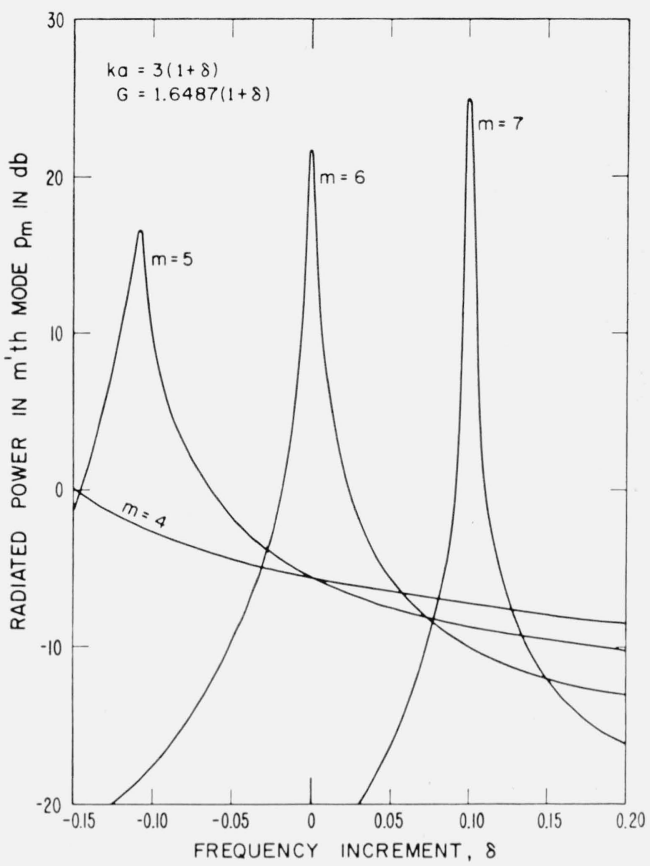

where $\epsilon_{0}=1, \epsilon_{m}=2$ for $m=2,3,4, \ldots . ., H_{m}^{(2)}(x)$ is a cylindrical Hankel function, $x=k a_{c}$ and $G$ is the normalized surface reactance. The radiated power $p_{m}$ in $\mathrm{db}$ is shown in figure 6 for $k a_{c}=3(1+\delta)$ and $G=1.649(1+\delta)$. This is the cylindrical analog to the resonance curves in figure 3 for the corrugated sphere. The similarity between these curves is rea lly quite striking. This provides a good check on the calculations since, from a physical standpoint, the resonance occurs from an interaction of peripheral surface waves.

\section{Conclusion}

On the basis of the results given here it is apparent that a spherical-shaped body, which has latitudinal corrugations, may be strongly resonated by a radial electric dipole located at the pole. It may be mentioned that the same state of affairs exists when the excitation is by a small annular slot which is also located at the pole.

We thank Mrs. Lillie Walters for her able assistance in carrying out this work.

\section{References}

Carter, P. S. (June 1932), Circuit relations in radiating systems and applications to antenna problems, Proc. IRE 20, No. 6, 1004-1041.

Cullen, A. L. (1960), Surface wave resonance effect in a reactive cylindrical structure excited by a line source, J. Res. NBS 64D (Radio Prop.), No. 1, 13-19.

Morse, P. M., and H. Feshbach (1953), Methods of theoretical physics (McGraw-Hill Book Co., New York, N.Y.).

Wait, J. R. (1962), Electromagnetic waves in stratified media (Pergamon Press, Oxford, England).

Wait, J. R., and A. M. Conda (Sept. 1960), The resonance excitation of a corrugated-cylinder antenna, Proc. Inst. Elec. Engrs. London Pt. C, 107, 362-366. (In figure 4 of this paper the conditions should be listed as $G=(1.6487+\delta)$ and $k a=(3+\delta)$.)

Zucker, F. J. (1961), Surface and leaky-wave antennas, ch. 16, Antenna Engineering Handbook, ed. H. Jasik (McGraw-Hill Book Co., New York, N.Y.).

(Paper 67D3-268) 